\title{
Femtosecond Spectroscopy of Molecular Autoionization and Fragmentation
}

\author{
T. Baumert, B. Bühler, R. Thalweiser, and G. Gerber \\ Fakultät für Physik, Universität Freiburg, D-7800 Freiburg, Federal Republic of Germany \\ (Received 6 November 1989)
}

\begin{abstract}
Femtosecond laser pulses are applied to the study of the dynamics and the pathways of multiphotoninduced ionization, autoionization, and fragmentation of $\mathrm{Na}_{2}$ in molecular-beam experiments. In particular, we report on first results obtained studying electronic autoionization (leading to $\mathrm{Na}_{2}{ }^{+}+e^{-}$) and autoionization-induced fragmentation (leading to $\mathrm{Na}^{+}+\mathrm{Na}^{+} e^{-}$) of a bound doubly excited molecular state. The final continuum states are analyzed by photoelectron spectroscopy and by measuring the mass and the released kinetic energy of the corresponding ionic fragments with a time-of-flight arrangement.
\end{abstract}

PACS numbers: $33.60 .-\mathrm{q}, 33.80 . \mathrm{Eh}$

In contrast to the well studied two-electron excited states of various atomic systems, the electronic autoionization of doubly excited molecular states is still a field where very little is known. Doubly excited states are crucial to the understanding of the dynamics of diatomic molecules at energies where electronic autoionization, ionic fragmentation, and neutral fragmentation compete. The study of the coupling of the different continua of fragmentation and ionization is also of great theoretical interest, because of the need for an extension of multichannel quantum-defect theory (MQDT) to treat doubly excited electronic states, dissociating states, and autoionizing Rydberg states together.

Interesting studies of the dissociation and autoionization of unbound doubly excited states in $\mathrm{H}_{2}$ have recently been reported by several groups. ${ }^{1}$ The first spectroscopic study of a bound doubly excited state, the ${ }^{1} \Sigma_{g}^{-}(3 d+4 p)$ state of $K_{2}$, has also only recently been reported. $^{2}$ However, there has yet been no report on the interaction of a particular bound doubly excited state with different continua and the competition between the various decay channels of such a state. Electronic autoionization and in particular autoionization-induced molecular fragmentation have been neither experimentally nor theoretically explored as much as the vibrational and rotational autoionization of Rydberg states. Beside experimental difficulties, the main reason for this lack of information and understanding is that often neither the final continuum states are analyzed nor the dissociative ionization clearly distinguished from neutral fragmentation with subsequent excited-fragment photoionization when using nanosecond or even picosecond laser pulses. Of course, both processes may lead to formation of the same ionic fragment. Femtosecond laser pulses offer an experimental tool fast enough to distinguish between these two processes. If the laser pulse durations applied are considerably shorter $(\tau \approx 100 \mathrm{fs})$ than the fragment separation time, no further laserinduced excitation or ionization of fragments can occur. For $\mathrm{Na}_{2}$, the time needed for the fragments to separate to $10 \AA$ varies from 0.5 to 1 ps depending on the recoil energy $W$. All observations therefore have to be related to excitation processes taking place at small internuclear distances.

Pioneering work in the field of femtosecond photochemistry and transition-state spectroscopy has been performed by Zewail and co-workers. ${ }^{3}$ In a series of beautiful experiments in the gas phase and in molecular beams, they have demonstrated the enormous advantage of applying femtosecond lasers to the study of molecular dynamics. $^{3}$

The multiphoton ionization and fragmentation of alkali-metal dimers and, in particular, of $\mathrm{Na}_{2}$ has been the subject of considerable current interest. In many experiments it has been found that in conjunction with the formation of $\mathrm{Na}_{2}{ }^{+}$ions, the atomic ions $\mathrm{Na}^{+}$are also produced. It is well established ${ }^{4}$ that resonance-enhanced multiphoton-ionization (REMP) processes via the $A^{1} \Sigma_{u}^{+}$or the $B^{1} \Pi_{u}$ states are responsible for this observation, whereas nonresonant multiphoton processes only play a minor role. Ionization and fragmentation of $\mathrm{Na}_{2}$ Rydberg states involving vibrational-rotational autoionization have also been studied in recent years. ${ }^{5}$ However, very few experiments have reported a clear identification of the fragmentation pathways. Two examples of such detailed studies are (i) the fragmentation of the neutral molecule $\mathrm{Na}_{2}{ }^{*}$ into $\mathrm{Na}^{*}(3 p)+\mathrm{Na}^{*}(3 p)$, i.e., the reverse process to laser-induced associative ionization, applying Doppler spectroscopy to measure the angular and energy distributions of the neutral fragments, ${ }^{6}$ and (ii) the two-photon ionization and dissociation of $\mathrm{Na}_{2}$ resonantly enhanced via the $(2)^{1} \Sigma_{u}^{+}$doubleminimum state with kinetic-energy analysis of the ionic fragments. ${ }^{7}$

To study the dynamics of multiphoton-induced excitation, autoionization, and fragmentation of $\mathrm{Na}_{2}$, we have used a combination of different experimental techniques. Femtosecond laser pulses are used to induce the transitions and time-of-flight (TOF) spectroscopy to determine both the mass and released kinetic energies of the fragments and the energies and angular distributions of ejected electrons. 


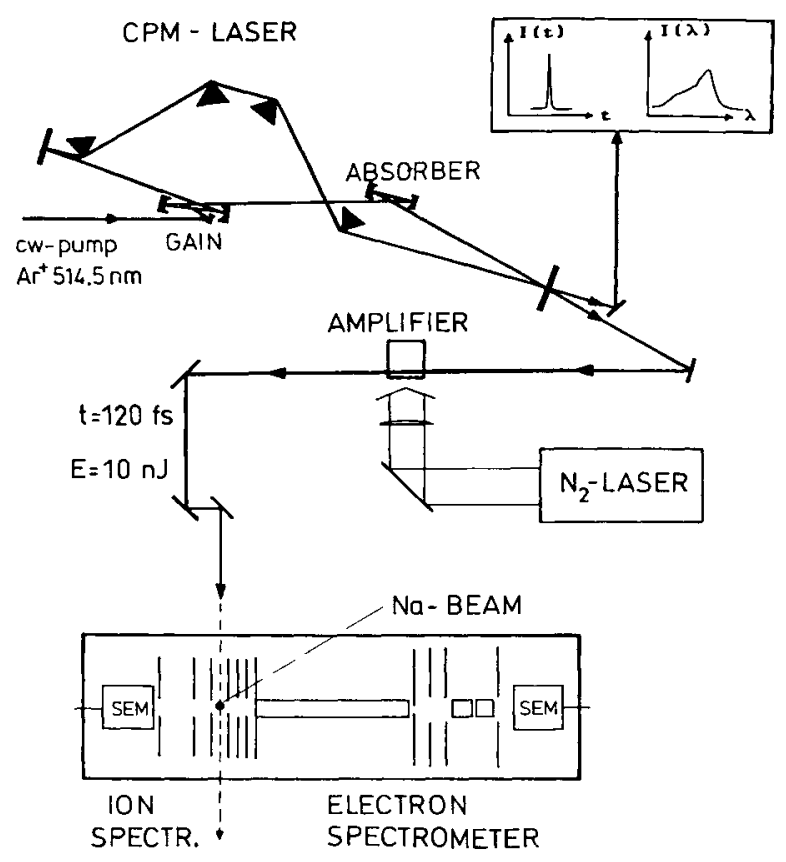

FIG. 1. Femtosecond-laser-molecular-beam arrangement. The molecular beam, the CPM laser, and the TOF axis are all perpendicular to each other.

Femtosecond pulses are generated in a home-built colliding-pulse mode-locked (CPM) ring dye laser. ${ }^{8}$ The emission peak of the CPM dye laser has been shifted to $616 \mathrm{~nm}$ by adjusting the diethyloxadicarbocyanine iodide (DODCI) absorber concentration. The spectral width (FWHM) was about $55 \AA$. The output of the CPM dye laser was amplified at a rate of $100 \mathrm{~Hz}$ in a $\mathrm{N}_{2}$ laser-pumped dye amplifier to produce pulses of $10 \mathrm{~nJ}$ energy and $t=120 \mathrm{fs}$ time duration measured by autocorrelation. The laser-pulse energy was intentionally kept at this low level. The laser system and the schematic experimental arrangement of the ion and electron time-of-flight spectrometers are shown in Fig. 1. The laser-molecular-beam interaction region is placed between parallel plates and since the ions are extracted perpendicular to the beam with a low electric field (10 $\mathrm{V} / \mathrm{cm}$ ), parent ions can be distinguished from fragment ions having initial kinetic energy. This experimental technique is widely known. ${ }^{9}$ The ion TOF spectrum displayed in Fig. 2(a) is obtained when our femtosecond laser $\left(I-10^{8} \mathrm{~W} / \mathrm{cm}^{2}\right)$ interacts with the sodium molecular beam. The spectrum clearly shows the observation of $\mathrm{Na}_{3}{ }^{+}, \mathrm{Na}_{2}{ }^{+}$, and "slow" $\mathrm{Na}^{+}$as well as "fast" $\mathrm{Na}^{+}$ ionic fragments resulting from the femtosecond laser excitation at $\lambda=616 \mathrm{~nm}$.

The small signal of the metal cluster $\mathrm{Na}_{3}{ }^{+}$is due to an absorption of two photons of $616 \mathrm{~nm}$, which are sufficient to ionize the $\mathrm{Na}_{3}$ cluster. ${ }^{10}$ The intense $\mathrm{Na}_{2}{ }^{+}$ signal is due to a REMPI process as is discussed below in detail. Predissociation of $\mathrm{Na}_{2}{ }^{*}$ and photoionization of $\mathrm{Na}^{*}$ as the origin of the observed $\mathrm{Na}^{+}$ions can be ruled out considering the time duration of our fs laser pulse.
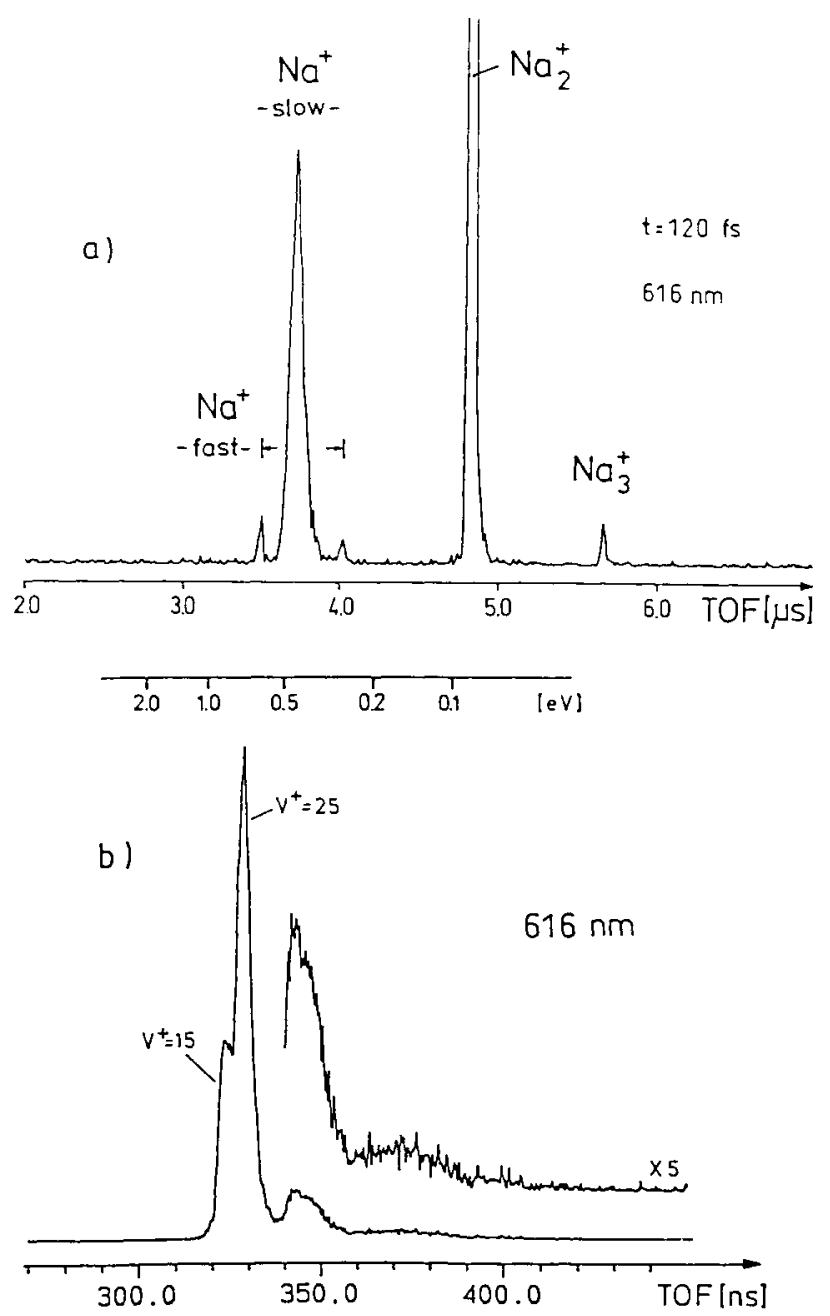

FIG. 2. (a) Time-of-flight spectrum of ions formed by the interaction of femtosecond laser pulses with $a^{\text {sodium }}$ molecular beam and (b) time-of-flight spectrum of ejected electrons.

Therefore the fast and slow $\mathrm{Na}^{+}$ions originate from fragmentation processes occurring at small internuclear distances of $\mathrm{Na}_{2}$. Based on this result, which is rather difficult to obtain from other experiments, and with the known molecular-potential curves, we completely determined for this model case the multiphoton excitation and fragmentation pathways.

The one-color REMPI spectrum between 610 and 620 $\mathrm{nm}$ obtained with a 2.5-ns pulsed tunable dye laser shows an isolated and strong peak at $616.08 \mathrm{~nm}$ in both the $\mathrm{Na}_{2}{ }^{+}$and the $\mathrm{Na}^{+}$channels. Based on the known spectroscopy of molecular Rydberg states of $\mathrm{Na}_{2},{ }^{11}$ it is evident that the peak in the $\mathrm{Na}_{2}{ }^{+}$spectrum is due to resonance-enhanced three-photon ionization of $\mathrm{Na}_{2}$ (Fig. 3). The ionization-enhancing intermediate electronic state ${ }^{1} \Pi_{g}(3 s+3 d)$ is populated by the two-photon process $\mathrm{Na}_{2}\left(X^{1} \Sigma_{g}^{+}, v^{\prime \prime}=0\right)+2 h v \rightarrow \mathrm{Na}_{2}\left(\operatorname{Ryd}^{1} \Pi_{g}, v^{*}=20\right)$. The Rydberg molecule is then photoionized by absorption of a third photon leading to the observed strong $\mathrm{Na}_{2}{ }^{+}$signal. This direct ionization leads to observed electrons having kinetic energies of $E=810 \pm 10 \mathrm{meV}$ 
and $E=940 \pm 10 \mathrm{meV}$. From this we conclude that the dimer ions are preferentially formed in the $v^{+}=24,25$ and $v^{+}=14,15$ vibrational states of the electronic ground state $X^{2} \Sigma_{g}^{+}$, which is in accord with the calculated Franck-Condon factors. Exactly the same excitation and ionization process happens when we use our femtosecond laser with its central wavelength of $616 \mathrm{~nm}$. Considering the predominant production of the $\mathrm{Na}_{2} \mathrm{~mol}$ - ecules in the $v^{\prime \prime}=0$ level in a supersonic molecular-beam expansion, the two-photon transition probability, and the upper ${ }^{1} \Pi_{g}$-Rydberg-state vibrational spacing $\Delta G \approx 88$ $\mathrm{cm}^{-1}$, we estimate that $90 \%$ of the excited $\mathrm{Na}_{2}$ molecules are in the $v^{*}=20$ level, despite the broad spectral distribution ( $\approx 140 \mathrm{~cm}^{-1} \mathrm{FWHM}$ ) of the fs laser pulse. The created dimer ions may now undergo a bound-free transition by absorption of one more photon from still the same fs laser pulse:

$$
\mathrm{Na}_{2}{ }^{+}\left(X^{2} \Sigma_{g}^{+}, v^{+}\right)+h v \rightarrow \mathrm{Na}_{2}{ }^{+*}\left({ }^{2} \Sigma_{u}^{+}\right) \rightarrow \mathrm{Na}{ }^{+}+\mathrm{Na}(3 s)+W .
$$

Taking into account the populated $v^{+}$levels and the known potential curves for the ionic ground and firstexcited states, ${ }^{12}$ the corresponding bound-free transitions lead to recoil energies $W$ between 9910 and 11037 $\mathrm{cm}^{-1}$. The energy obtained from the analysis of the ion TOF spectrum, namely, $W=10500 \pm 500 \mathrm{~cm}^{-1}$, surely agrees with this prediction. We therefore conclude on the basis of the observed ion and electron kinetic energies (Fig. 2) and the restriction imposed by using 120-fs laser pulses that fast $\mathrm{Na}^{+}$ions are produced by the ionization and fragmentation process shown in Fig. 3.

The observation of slow $\mathrm{Na}^{+}$ions, however, cannot be explained within this framework since photoionization of the vibrational level $v^{*}=20$ of the ${ }^{1} \Pi_{g}$ Rydberg state directly into the ${ }^{2} \Sigma_{u}^{+}$continuum is energetically forbidden for the applied laser wavelength. The electron spectrum in Fig. 2(b) shows in addition to the two strong peaks less intense broad structures in the range between 300 and $500 \mathrm{meV}$ and around $160 \mathrm{meV}$ extending to

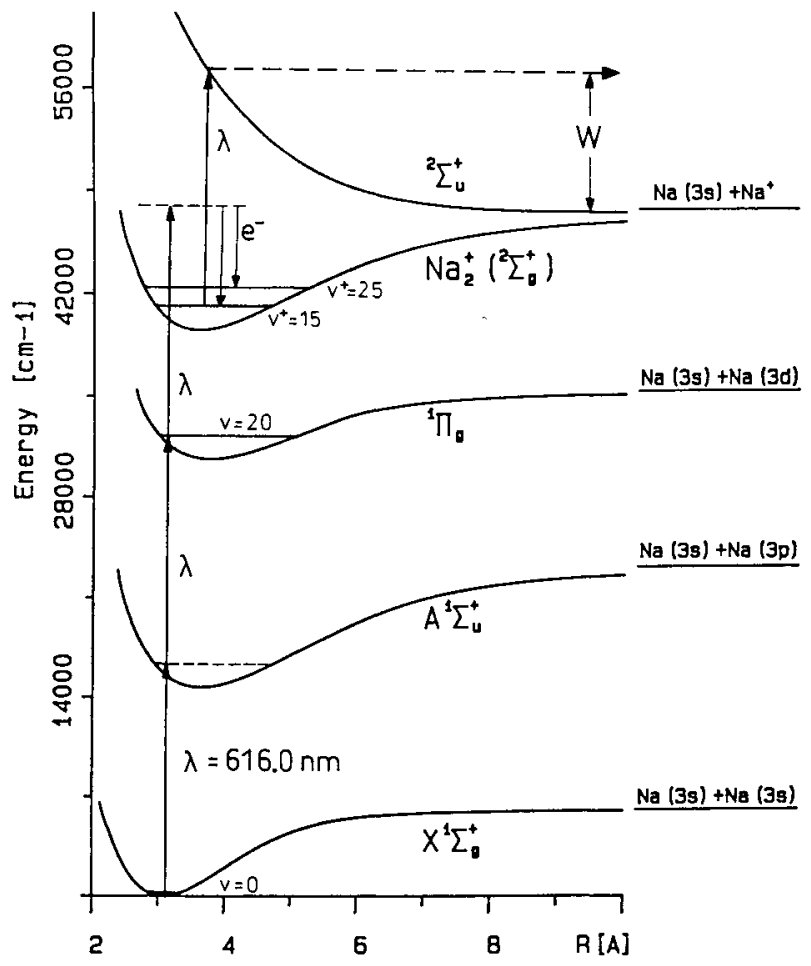

FIG. 3. Potential-energy diagram of $\mathrm{Na}_{2}$ illustrating the process leading to fast $\mathrm{Na}^{+}$ionic fragments. very low energies. Based on both the measured electron energy distribution and the distribution of recoil energies $W=900 \pm 400 \mathrm{~cm}^{-1}$, obtained from the analysis of the slow $\mathrm{Na}^{+}$ionic fragments, a consistent interpretation is found by considering the excitation and the decay of doubly excited molecular states of $\mathrm{Na}_{2}$. Figure 4 shows theoretical $^{12}$ potential curves of the ground state ${ }^{2} \Sigma_{g}^{+}$ and the repulsive state ${ }^{2} \Sigma_{u}^{+}$of the $\mathrm{Na}_{2}{ }^{+}$ion and a schematic potential curve of the doubly excited ${ }^{1} \Pi_{u}(4 s+3 p)$ state of $\mathrm{Na}_{2}{ }^{* *}$ (dashed curve). There is no other asymptotic limit of the bound doubly excited state in that energy range. The doubly excited states $\mathrm{Na}_{2}{ }^{* *}(n l+3 p)$ form a Rydberg series converging to the ${ }^{2} \Pi_{u}$ state of $\mathrm{Na}_{2}{ }^{+}$, whose potential curve is known

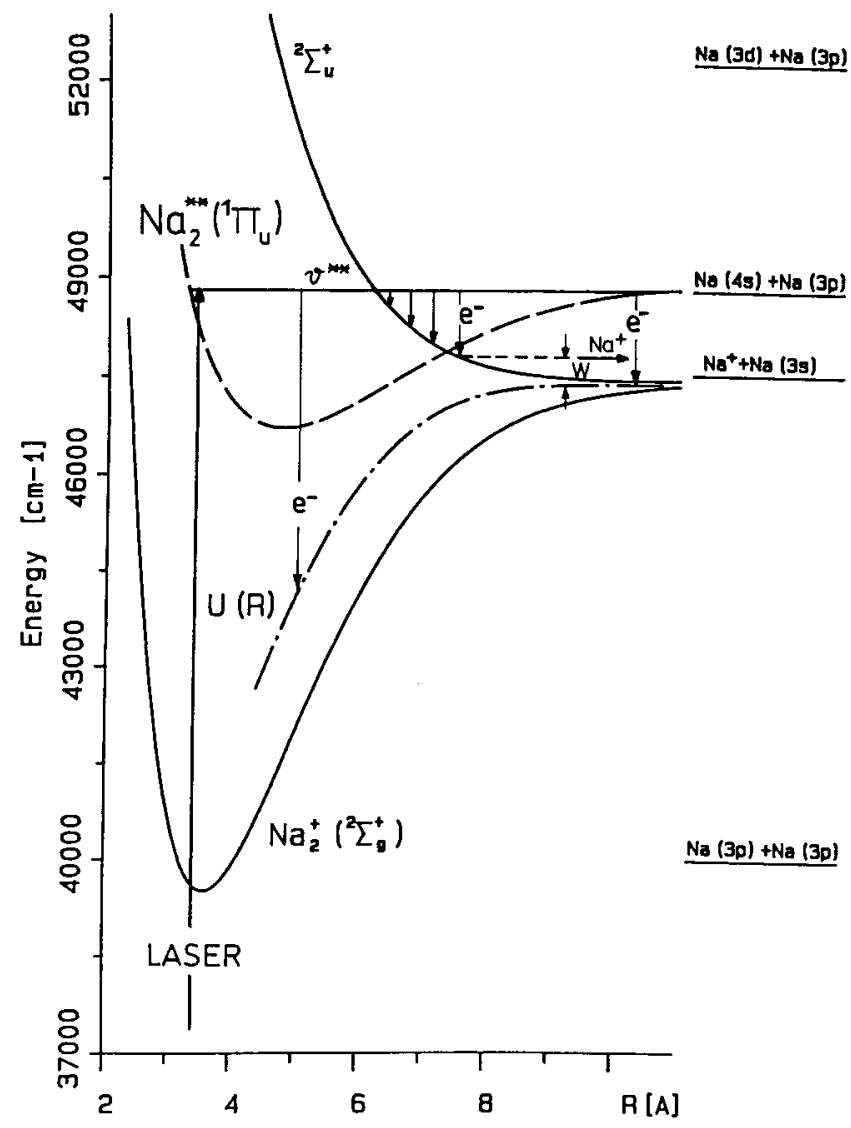

FIG. 4. Excitation and autoionization processes of the doubly excited ' $\Pi_{u}(4 s+3 p)$ state of $\mathrm{Na}_{2}$. 
theoretically. ${ }^{12}$ Assuming that the shape of a ${ }^{1} \Pi_{u}$-state potential curve, correlated to $\mathrm{Na}(4 s)+\mathrm{Na}(3 p)$, is similar to the ionic ${ }^{2} \Pi_{u}$ curve and that it has a small potential barrier at large internuclear distances like the $B^{1} \Pi_{u}$ state built from $\mathrm{Na}(3 s)+\mathrm{Na}(3 p)$, we conclude that in the resonance-enhanced three-photon process vibronic levels close to the dissociation limit of the ${ }^{1} \Pi_{u}(4 s+3 p)$ state are excited. The wave functions of these vibronic levels extend from $3 \AA$ to $-10 \AA$. These doubly excited levels may now electronically autoionize into the $X^{2} \Sigma_{g}^{+}$ ground state of $\mathrm{Na}_{2}{ }^{+}$. Taking into account Mulliken's difference potential $U(R),{ }^{13}$ we expect for this particu- lar process ejected electrons with energies between 260 and $500 \mathrm{meV}$. These electrons are definitely observed. For internuclear distances greater than $6 \AA$, the vibronic levels of this doubly excited state interact additionally with the continuum of the repulsive ${ }^{2} \Sigma_{u}^{+}$state of $\mathrm{Na}_{2}{ }^{+}$. Therefore for $R \gtrsim 6 \AA$ a second channel is open for electronic autoionization. The autoionization process

$$
\mathrm{Na}_{2}^{* *}\left({ }^{1} \Pi_{u}(4 s+3 p)\right) \rightarrow \mathrm{Na}_{2}{ }^{+*}\left({ }^{2} \Sigma_{u}^{+}\right)+e^{-(}\left(E_{\mathrm{kin}}\right)
$$

is responsible for the observed electrons having energies in the range from 0 to $160 \mathrm{meV}$. The autoionizationinduced fragmentation

$$
\mathrm{Na}_{2}{ }^{* *}\left({ }^{1} \Pi_{u}(4 s+3 p)\right) \rightarrow \mathrm{Na}_{2}{ }^{* *}\left({ }^{2} \Sigma_{u}^{+}\right)+e^{-}\left(E_{\mathrm{kin}}\right) \rightarrow \mathrm{Na}{ }^{+}+\mathrm{Na}(3 s)+W
$$

leads to slow $\mathrm{Na}^{+}$ions whose kinetic energies depend on the internuclear distance $R$ where the autoionization takes place. A calculation of the autoionization electron spectrum arising from bound-free transitions ${ }^{1} \Pi_{u}(4 s+3 p) \rightarrow{ }^{2} \Sigma_{u}^{+}$shows essentially a broad distribution in agreement with the experimental electron energy spectrum. The observed range of released kinetic energies $W$ of the slow $\mathrm{Na}^{+}$fragment ions is also in accord. This example of autoionization and autoionizationinduced fragmentation shows that a unique assignment can only be given when the final continuum states are determined by measurements of the kinetic energy of the electrons and of the ionic fragments.

In conclusion, this is the first study where, in a molecular-beam experiment, a femtosecond laser is used in combination with ion and electron spectroscopy to clarify the ionization, autoionization, and fragmentation mechanisms of molecules excited by multiphoton processes into the energy region of doubly excited electronic states. The electronic autoionization of the ${ }^{1} \Pi_{u}(4 s+3 p)$ bound state of $\mathrm{Na}_{2}$ into the two ion continua $\left({ }^{2} \Sigma_{g}^{+}\right.$and ${ }^{2} \Sigma_{u}^{+}$of $\mathrm{Na}_{2}{ }^{+}$) and the autoionization-induced fragmentation are processes which have not been observed before and which undoubtedly enhance our knowledge of doubly excited molecular states.

This work has been supported by the Deutsche Forschungsgemeinschaft, Sonderforschungsbereich No. 276.

IJ. W. Verschuur and H. B. van Linden ven den Heuvell, Chem. Phys. 129, 1 (1989), S. T. Pratt, P. M. Dehmer, and J.
L. Dehmer, J. Chem. Phys. 86, 1727 (1987); M. A. O’Halloran et al., J. Chem Phys. 87, 3288 (1987).

${ }^{2}$ A. Katern, P. Kowalczyk, and F. Engelke, Chem. Phys. Lett. 146, 325 (1988).

${ }^{3}$ M. Rosker, M. Dantus, and A. H. Zewail, J. Chem. Phys. 89, 6113 (1988), and references therein; T. S. Rose, M. J. Rosker, and A. H. Zewail, J. Chem. Phys. 88, 6672 (1988); and references therein.

${ }^{4}$ J. Keller and J. Weiner, Phys. Rev. A 30, 213 (1984); C. E. Burkhardt, W. P. Garver, and J. J. Leventhal, Phys. Rev. A 31, 505 (1985); F. Roussel, P. Breger, and G. Spiess, J. Phys. B 18 , 3769 (1985).

${ }^{5}$ C. Bordas et al., Phys. Rev. Lett. 60, 917 (1988); P. Labastie et al., Mol. Phys. 59, 29 (1986); C. Bordas et al., Chem. Phys. 129, 21 (1989); R. Haugstätter, A. Goerke, and I. V. Hertel, Phys. Rev. A 39, 5084 (1989).

${ }^{6}$ G. Gerber and R. Möller, Phys. Rev. Lett. 55, 814 (1985); in Photons and Continuum States of Atoms and Molecules, edited by N. K. Rahman, C. Guidotti, and M. Allegrini, Springer Proceedings in Physics Vol. 16 (Springer-Verlag, Berlin, 1987), p. 126.

${ }^{7}$ R. Haugstätter, A. Goerke, and I. V. Hertel, Z. Phys. D 9, 153 (1988).

${ }^{8}$ J. A. Valdmanis and R. L. Fork, IEEE J. Quantum Electron. 22, 112 (1986).

${ }^{9}$ See, for example, R. Ogorzalek Loo, G. E. Hall, H. P. Härri, and P. L. Houston, J. Phys. Chem. 92, 5 (1988).

${ }^{10} \mathrm{M}$. Broyer et al., Z. Phys. D 3, 131 (1986).

${ }^{\prime \prime}$ N. W. Carlson et al., Phys. Rev. A 24, 822 (1981); C. Bordas et al., Chem. Phys. 129, 21 (1989).

${ }^{12}$ W. Müller and W. Meyer, J. Chem. Phys. 80, 3311 (1984); thesis, I. Schmidt Universität, Kaiserslautern, 1987 (unpublished).

${ }^{13}$ R. S. Mulliken, J. Chem. Phys. 55, 309 (1971). 